\title{
Diagnosis of Wunderlich syndrome in a patient with flank pain
}

YY Lin, CW Hsu, HM Li, HY Su *

Hong Kong Med J 2019;25:406.e1-2

https://doi.org/10.12809/hkmj187718

In September 2018, a 62-year-old man without underlying disease presented to the emergency department of E-Da Hospital, Kaohsiung, Taiwan, with right flank pain for 1 day. The patient reported sharp and persistent pain radiating to the right upper abdomen. On arrival at the emergency department, the patient had heart rate 120 beats per minute and blood pressure $85 / 54 \mathrm{~mm} \mathrm{Hg}$. Physical examination revealed right flank knocking tenderness. Laboratory test results, including blood test and urinary analysis, were unremarkable. Abdominal plain film radiograph revealed a large right renal mass displacing surrounding structures (Fig 1). Point-of-care ultrasound demonstrated a right renal mass with hyperechogenicity, which was surrounded by hypoechoic haematoma in the perinephric space (Fig 2). Subsequent abdominal computed tomography $(\mathrm{CT})$ revealed rupture of right renal angiomyolipoma with pericapsular haematoma (Fig 3). Wunderlich syndrome complicated by hypovolaemic shock was diagnosed, and proper fluid resuscitation and blood transfusion were performed in the emergency department. The patient received partial nephrectomy of right kidney on the next day, and was discharged uneventfully from the hospital 2 weeks after admission.

Wunderlich syndrome, a rare but lifethreatening entity, is defined as spontaneous nontraumatic renal haemorrhage confined to the subcapsular and perirenal space. ${ }^{1}$ Lenk's triad, which consists of acute flank pain, palpable flank mass, and hypovolemic shock, is the classical clinical feature of Wunderlich syndrome. ${ }^{2}$ The aetiologies of Wunderlich syndrome are classified into neoplastic and non-neoplastic origins. Up to $60 \%$ of patients with Wunderlich syndrome are caused by neoplasm, including benign tumours such as angiomyolipoma and malignancies such as renal cell carcinoma. ${ }^{3} \mathrm{~A}$ variety of diseases account for non-neoplastic origins of Wunderlich syndrome, including vasculitis, renal artery aneurysm, arteriovenous malformation, renal vein thrombosis, nephritis, cystic renal disease, and coagulopathy. ${ }^{3}$ Angiomyolipoma, a benign neoplasm composed of smooth muscle, adipose tissue, and thick-walled blood vessels, is the most common cause of Wunderlich syndrome. ${ }^{3}$ The risk of tumour rupture leading to fatal internal haemorrhage increases when angiomyolipoma grows $>40 \mathrm{~mm}$ in diameter. ${ }^{4}$ Aneurism formation due to poor elastic vascular structure might be the reason for

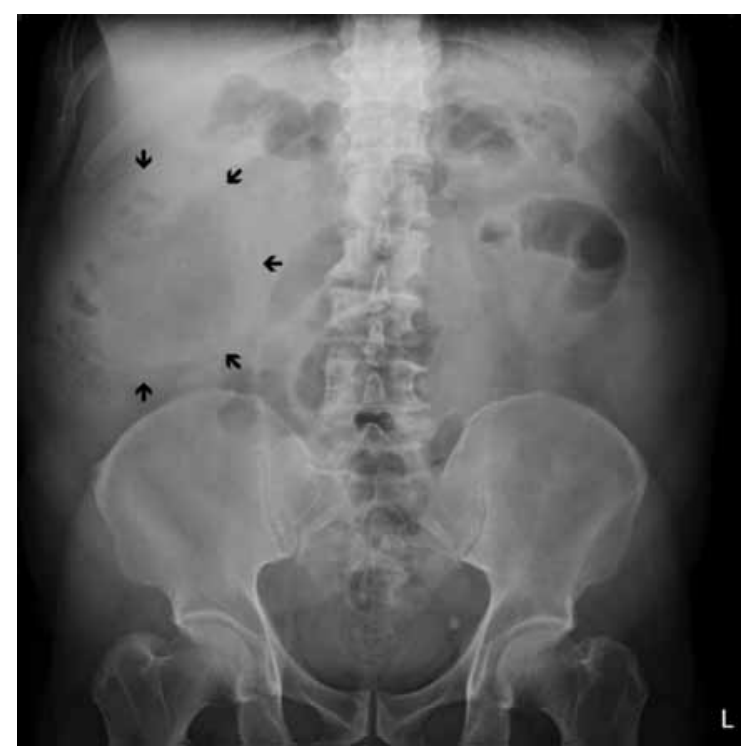

FIG I. Plain abdominal radiograph showing a large right renal mass displacing surrounding structure (arrows). The low density of the mass is suggestive of a lesion with a lipomatous component

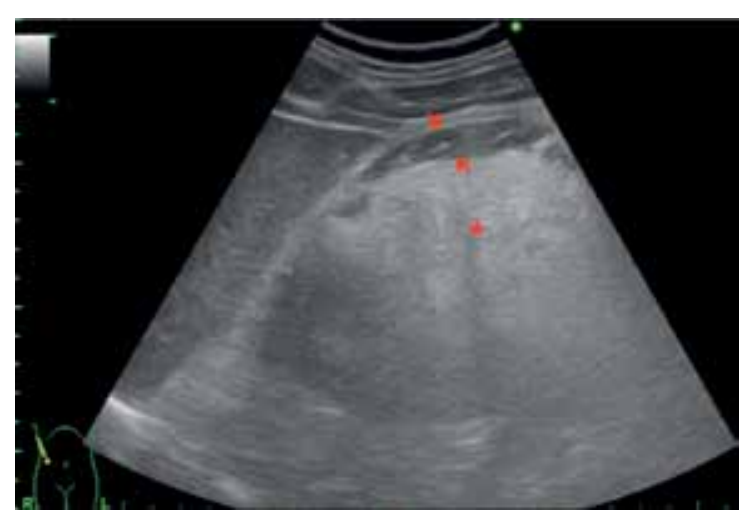

FIG 2. Point-of-care ultrasound showing hypoechoic haematoma in the perinephric space (arrows).

Hyperechogenicity can indicate a lipomatous component such as angiomyolipoma in the kidney (star)

angiomyolipoma rupture, especially during tumour growth.

For diagnosis of Wunderlich syndrome, contrast-enhanced CT scan is a standard medical imaging modality with $100 \%$ sensitivity in identifying perirenal haemorrhage. ${ }^{4}$ Computed tomography 


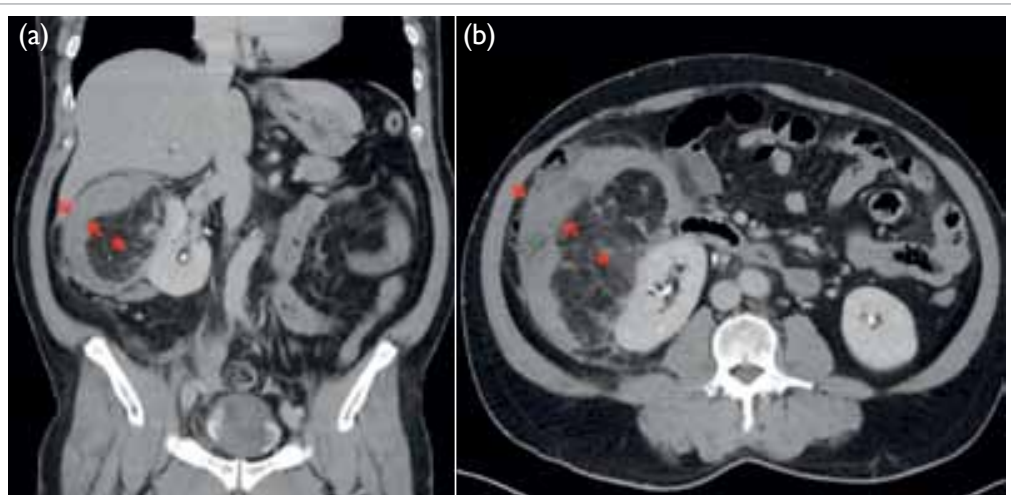

FIG 3. (a) Coronal and (b) axial contrast-enhanced computed tomography images showing rupture of a right renal angiomyolipoma (stars) with pericapsular haematoma (arrows) presenting with flank pain and in shock to facilitate timely emergency surgery or embolisation if needed.

\section{Author contributions}

All authors had full access to the data, contributed to the study, approved the final version for publication, and take responsibility for its accuracy and integrity.

Concept and design of the study: HY Su.

Acquisition of data: YY Lin.

Analysis or interpretation of data: YY Lin.

Drafting of the article: HY Su.

Critical revision for important intellectual content: $\mathrm{HM} \mathrm{Li}$, CW Hsu.

\section{Conflicts of interest}

All authors have disclosed no conflicts of interest.

\section{Funding/support}

This study received no specific grant from any funding agency in the public, commercial, or not-for-profit sectors.

scan can present renal vascular structure, origins of tumours and pathological change in adjacent tissues. Furthermore, CT scan can also provide detailed vascular anatomy to provide a roadmap for superselective renal embolisation in management of perirenal haemorrhage. In contrast with $\mathrm{CT}$ scan, point-of-care ultrasound might be considered as a prompt tool to diagnose patients with Wunderlich syndrome. Point-of-care ultrasound can be used to screen the renal structure, quickly identify internal bleeding, and evaluate the hemodynamic condition by measuring the diameter of the inferior vena cava and assessing the cardiac preload and contractility. Ultrasound can also facilitate the initial differential diagnosis of patients with flank pain, such as renal colic, renal abscess or acute pyelonephritis. Initial treatments for Wunderlich syndrome include selective arterial embolisation and surgical intervention. However, clinical guidelines for management of Wunderlich syndrome are not yet well established. ${ }^{5}$ Selective arterial embolisation has the advantage of minimal invasiveness, renal preservation, and efficiency in treating acute renal haemorrhage. However, surgical intervention can provide a delicate strategy for tumour resection, especially if suspicious for malignancy, and prevent recurrent tumour bleeding. ${ }^{5}$ Since Wunderlich syndrome is a life-threatening condition, clinicians should be aware while approaching patients

\section{Ethics approval}

This study was conducted in accordance with the principles outlined in the Declaration of Helsinki.

YY Lin, MD

$\mathrm{HM} \mathrm{Li}, \mathrm{MD}$

HY Su *, MD

Department of Emergency Medicine, E-Da Hospital, I-Shou University, Kaohsiung, Taiwan

* Corresponding author: hys927@hotmail.com

\section{References}

1. Medda M, Picozzi SC, Bozzini G, Carmignani L. Wunderlich's syndrome and hemorrhagic shock. J Emerg Trauma Shock 2009;2:203-5.

2. Simkins A, Maiti A, Cherian SV. Wunderlich syndrome. Am J Med 2017;130:e217-8.

3. Katabathina VS, Katre R, Prasad SR, Surabhi VR, Shanbhogue AK, Sunnapwar A. Wunderlich syndrome: cross-sectional imaging review. J Comput Assist Tomogr 2011;35:425-33.

4. Albi G, del Campo L, Tagarro D. Wünderlich's syndrome: causes, diagnosis and radiological management. Clin Radiol 2002;57:840-5.

5. Flum AS, Hamoui N, Said MA, et al. Update on the diagnosis and management of renal angiomyolipoma. J Urol 2016;195(4 Pt 1):834-46.
CW Hsu, PhD 